\title{
A generalization of Perron's theorem about Hurwitzian numbers
}

\author{
by
}

Pierre Stambul (Marseille)

1. Introduction. Continued fractions of the form

$$
\left[c_{0} ; c_{1}, \ldots, c_{n}, \overline{Q_{1}(k), \ldots, Q_{p}(k)}\right]_{k=0}^{\infty}
$$

are called Hurwitzian if $c_{0}$ is an integer, $c_{1}, \ldots, c_{n}$ are positive integers, $Q_{1}, \ldots, Q_{p}$ are polynomials with rational coefficients which take positive integral values for $k=0,1,2, \ldots$ and at least one of the polynomials is not constant. $Q_{1}, \ldots, Q_{p}$ are said to form a quasi-period. The expansions

$$
e=[2, \overline{1,2 k+2,1}]_{k=0}^{\infty} \quad \text { and } \quad e^{1 / q}=[\overline{1,(2 k+1) q-1,1}]_{k=0}^{\infty}
$$

when $q$ is an integer $\geq 2$ are well-known examples (see Euler [3], Perron [7], Davis [2], Matthews and Walters [6]). Other classical examples of Hurwitzian numbers are $\operatorname{th}(1 / q)$ or $\tan (1 / q)$ when $q$ is a nonnegative integer, $e^{2 / q}$ when $q$ is odd and many other real numbers determined by means of Bessel functions (see Cabannes [1], Lehmer [4] and Stambul [10]). A recognizable Hurwitzian number whose quasi-period is determined by polynomials of degree $\geq 2$ is still unknown.

Let $h: x \mapsto(a x+b) /(c x+d)$ be a Möbius transformation where $a, b, c, d$ are integers. If $x$ is Hurwitzian, it follows from a theorem of $\mathrm{O}$. Perron $([7], 127-131)$ that $h(x)$ is also Hurwitzian. Moreover, the nonconstant polynomials in the quasi-periods of $x$ and $h(x)$ have the same degrees (see $[10])$.

Denote by $\mathcal{R}$ the set of all irrational real numbers $x$ whose continued fraction expansion has the form

$$
x=\left[c_{0} ; c_{1}, \ldots, c_{n-1}, \overline{C_{1}(k), \ldots, C_{p}(k)}\right]_{k=0}^{\infty}
$$

where the $\mathbb{N}^{p}$-valued sequence $k \mapsto\left(C_{1}(k), \ldots, C_{p}(k)\right)$ satisfies a linear homogeneous recurrence relation with constant coefficients in $\mathbb{Z}$, i.e., there exists a given $p \times p$ matrix $M$ with integer entries such that 


$$
\left(\begin{array}{c}
C_{p}(k+1) \\
C_{p-1}(k+1) \\
\vdots \\
C_{1}(k+1)
\end{array}\right)=M\left(\begin{array}{c}
C_{p}(k) \\
C_{p-1}(k) \\
\vdots \\
C_{1}(k)
\end{array}\right)
$$

for all integers $k \geq 0$.

The $C_{i}$ are "generalized power sums" (the restriction of exponential polynomials to the nonnegative integers) and $\left(C_{1}, \ldots, C_{p}\right)$ will be called a quasiperiod of $x$ (it is not unique). Irrational quadratic numbers and Hurwitzian numbers form subsets of $\mathcal{R}$. This result can be easily derived from the identity

$$
\sum_{i=0}^{r}(-1)^{i}\left(\begin{array}{l}
r \\
i
\end{array}\right) Q(x+i)=0
$$

for all real polynomials $Q$ of degree $\leq r-1$. Unfortunately, a recognizable number in $\mathcal{R}$ which is not in these subsets is still unknown.

Perron's proof is based on congruences and successive derivations of polynomials. In this paper, we generalize the result of Perron by means of a transducer: for all homographies $h$ with integral coefficients, $h(\mathcal{R}) \subset \mathcal{R}$.

2. Image by a Möbius transformation. Let $\xi$ be an irrational real number and $h: x \mapsto(a x+b) /(c x+d)$ a Möbius transformation where $a, b, c, d$ are integers. The continued fraction expansion of $h(\xi)$ has been studied by Raney [9], van der Poorten [8], Liardet and Stambul [5].

We recall basic definitions and facts given in [5], following [9]. A matrix $M=\left(\begin{array}{ll}\alpha & \beta \\ \gamma & \delta\end{array}\right)$ where $\alpha, \beta, \gamma, \delta$ are nonnegative integers and $(\alpha-\gamma)(\beta-\delta)<0$ is said to be row-balanced. All computations for the continued fraction expansion of $h(\xi)$ can be reduced to the case where $A=\left(\begin{array}{ll}a & b \\ c & d\end{array}\right)$ is rowbalanced and $\xi>1$. This computation is given by a finite state transducer $\mathcal{T}_{\mathcal{D}}=(\mathcal{C}, \mathcal{B}, \mathcal{A}, \Phi, \Psi)$ where

- The input alphabet is $\mathcal{C}=\mathbb{N} \backslash\{0\}$.

- The space of states $\mathcal{B}$ is the set of all row-balanced matrices $M$ such that $|\operatorname{det}(M)|=|\operatorname{det}(A)|=D$. Clearly, $\mathcal{B}$ is a finite set. The initial state of the transducer is $A$.

- The output alphabet is $\mathcal{A}=\mathbb{N}$ and the monoid generated by $\mathcal{A}$ is denoted by $\mathcal{A}^{*}$.

It is well known that every $2 \times 2$ matrix $M$ of rank 2 with nonnegative entries that is not row-balanced can be written in a unique way as

$$
M=\left(\begin{array}{cc}
a_{0} & 1 \\
1 & 0
\end{array}\right)\left(\begin{array}{cc}
a_{1} & 1 \\
1 & 0
\end{array}\right) \ldots\left(\begin{array}{cc}
a_{n} & 1 \\
1 & 0
\end{array}\right) M^{\prime}
$$

where $M^{\prime}$ is row-balanced, $a_{0} \in \mathbb{N}$ and $a_{1}, \ldots, a_{n} \in \mathbb{N} \backslash\{0\}$. 
- $\Phi=\left\{\phi_{c}: c \in \mathcal{C}\right\}$ and $\Psi=\left\{\psi_{c}: c \in \mathcal{C}\right\}$ are two families of maps $\left(\phi_{c}: \mathcal{B} \rightarrow \mathcal{B}\right.$ and $\left.\psi_{c}: \mathcal{B} \rightarrow \mathcal{A}^{*}\right)$ defined as follows: for all $B$ in $\mathcal{B}$ and $c$ in $\mathcal{C}$, if $B\left(\begin{array}{ll}c & 1 \\ 1 & 0\end{array}\right)=B^{\prime}$ is row-balanced, then $\phi_{c}(B)=B^{\prime}$ and $\psi_{c}(B)=\wedge$ (the empty word). And if $B\left(\begin{array}{ll}c & 1 \\ 1 & 0\end{array}\right)$ is not row-balanced, then by $(\mathrm{F})$,

$$
B\left(\begin{array}{ll}
c & 1 \\
1 & 0
\end{array}\right)=\left(\begin{array}{cc}
a_{0} & 1 \\
1 & 0
\end{array}\right)\left(\begin{array}{cc}
a_{1} & 1 \\
1 & 0
\end{array}\right) \ldots\left(\begin{array}{cc}
a_{n} & 1 \\
1 & 0
\end{array}\right) B^{\prime} .
$$

Then $\phi_{c}(B)=B^{\prime}$ and $\psi_{c}(B)=a_{0} a_{1} \ldots a_{n}$.

Now, with any input word $c_{0} c_{1} \ldots c_{k}$ in $\mathcal{C}^{k}$, we associate a sequence of states $B_{1}=\phi_{c_{0}}(A)$ and $B_{i+1}=\phi_{c_{i}}\left(B_{i}\right), i=1, \ldots, k$, and we define

$$
[\Psi, \Phi]_{c_{0} c_{1} \ldots c_{k}}=\psi_{c_{0}}(A) \psi_{c_{1}}\left(B_{1}\right) \ldots \psi_{c_{k}}\left(B_{k}\right),
$$

which is a word in $\mathcal{A}^{*}$.

Finally, let $\mu$ be the "contraction map" which transforms a word in $\mathcal{A}^{*}$ into a word where all letters are positive integers (except maybe the first one), replacing from left to right subwords $a 0 b \quad(a, b \neq 0)$ by the letter $a+b$. A theorem of [5] shows that if $\xi=\left[c_{0} ; c_{1}, \ldots, c_{k}, \ldots\right]$ and $\mu \circ[\Psi, \Phi]_{c_{0} c_{1} \ldots c_{k}}=$ $a_{0} a_{1} \ldots a_{n-1} a_{n}$, then $h(\xi)=\left[a_{0} ; a_{1}, \ldots, a_{n-1}, \ldots\right]$ and the partial quotient following $a_{n-1}$ is $\geq a_{n}$.

Theorem. Let $\xi$ be in $\mathcal{R}$ and $h: x \mapsto(a x+b) /(c x+d)$ be a Möbius transformation where $a, b, c, d$ are integers. Then $h(\xi) \in \mathcal{R}$.

The proof requires three technical lemmas.

Lemma 1. Let $a, b, c, d$ be integers such that $|a d-b c|=D \geq 2$. Suppose that $\xi \in \mathcal{R}$ and compute $(a \xi+b) /(c \xi+d)$ with the transducer $\mathcal{T}_{\mathcal{D}}$. Then there exists two integers $r$ and $q$ such that the continued fraction expansion of $\xi$ has the form

$$
\xi=\left[c_{0} ; c_{1}, \ldots, c_{r-1}, \overline{A_{1}(k), \ldots, A_{q}(k)}\right]_{k=0}^{\infty}
$$

with the following properties:

(i) $A_{j}(k+1)=A_{j}(k) \bmod D$ for $j=1, \ldots, q$ and for all integers $k \geq 0$.

(ii) Either $A_{j}(k)>D$ for every integer $k \geq 0$, or the sequence $A_{j}(k)_{k \in \mathbb{N}}$ is constant.

(iii) By applying the transducer $\mathcal{T}_{D}$, the sequence of states $\left(B_{m}\right)_{m \in \mathbb{N}}$ satisfies $B_{r}=B_{r+q}$.

P r o o f. It is well known that every sequence of integers given by a linear recurrence relation with coefficients in $\mathbb{Z}$ is ultimately periodic modulo $D$ for all positive integers $D$. Hence (i) is obvious. Moreover, by the SkolemMahler theorem, the set of zeros of a linear recursive sequence $\left(u_{n}\right)_{n \in \mathbb{N}}$ with integral coefficients is equal to the union of a finite set and a finite number of arithmetical progressions. Clearly, the set of all integers $n$ such that $u_{n}=c$ for a given integer $c$ has the same property. 
Thus every number $\xi$ in $\mathcal{R}$ can be defined by a continued fraction expansion $\left[c_{0} ; c_{1}, \ldots, c_{n-1}, \overline{C_{1}(k), \ldots, C_{p}(k)}\right]_{k=0}^{\infty}$ where all the sequences $\left(C_{i}(k)\right)_{k \in \mathbb{N}}$ are either ultimately periodic or $>D$ for $k$ sufficiently large. Replacing $p$ by $p_{1}=s p \quad$ ( $s$ is a common multiple of all periods of periodic sequences $\left.C_{i}(k)\right), \xi$ has a continued fraction expansion of the form $\left[c_{0} ; c_{1}, \ldots, c_{n^{\prime}}, \overline{A_{1}^{\prime}(k), \ldots, A_{p_{1}}^{\prime}(k)}\right]_{k=0}^{\infty}$ with a quasi-period which satisfies (i) and (ii). Finally, by applying the transducer $\mathcal{T}_{D}$, let us consider the sequence of states $\left(B_{n^{\prime}+m p_{1}}\right)_{m \in \mathbb{N}}$. As $\mathcal{B}$ is a finite set, there exist two nonnegative integers $m_{1}$ and $m_{2}\left(m_{1}<m_{2}\right)$ such that $B_{n^{\prime}+m_{1} p_{1}}=B_{n^{\prime}+m_{2} p_{1}}$. Put $q=\left(m_{2}-m_{1}\right) p_{1}$; then $\xi$ is defined by a quasi-period of length $q$ which has the three properties.

Lemma 2. Let $B$ be a row-balanced matrix such that $|\operatorname{det}(B)|=D, c$ be a given integer $>D$ and $\left(f_{k}\right)_{k \geq 0}$ be a sequence of nonnegative integers. Then, for all integers $k \geq 0$, by applying $(\mathrm{F})$,

$$
B\left(\begin{array}{cc}
c+D f_{k} & 1 \\
1 & 0
\end{array}\right)=C\left(\begin{array}{cc}
f_{k}^{\prime} & 1 \\
1 & 0
\end{array}\right) B^{\prime}
$$

with the following properties:

- $C$ and $B^{\prime}$ are independent of $f_{k}$.

- $\operatorname{det}(C)= \pm 1$, i.e. either $C$ is the identity and has to be cancelled, or $C$ can be written in a unique way as

$$
C=\left(\begin{array}{cc}
a_{0} & 1 \\
1 & 0
\end{array}\right)\left(\begin{array}{cc}
a_{1} & 1 \\
1 & 0
\end{array}\right) \ldots\left(\begin{array}{cc}
a_{n} & 1 \\
1 & 0
\end{array}\right) \quad \text { with } a_{0} \in \mathbb{N}, a_{1}, \ldots, a_{n} \in \mathbb{N} \backslash\{0\} .
$$

- $f_{k}^{\prime}$ is the image of $c+D f_{k}$ by a map $x \mapsto(u x+v) / w$ with integral coefficients which depend on $B, D$ and $c$.

- $B^{\prime}$ is a row-balanced matrix and $\operatorname{det}\left(B^{\prime}\right)=D$.

Pro of. We distinguish four cases for the matrix $B=\left(\begin{array}{cc}\alpha & \beta \\ \gamma & \delta\end{array}\right)$.

1. $\alpha=0$. Then $\beta \gamma=D, \beta>\delta$ and

$$
\left(\begin{array}{ll}
0 & \beta \\
\gamma & \delta
\end{array}\right)\left(\begin{array}{cc}
c+D f_{k} & 1 \\
1 & 0
\end{array}\right)=\left(\begin{array}{cc}
0 & 1 \\
1 & 0
\end{array}\right)\left(\begin{array}{cc}
f_{k}^{\prime} & 1 \\
1 & 0
\end{array}\right)\left(\begin{array}{ll}
\beta & 0 \\
\phi & \gamma
\end{array}\right)
$$

with

$$
f_{k}^{\prime}=\gamma^{2} f_{k}+\left[\frac{\gamma c+\delta}{\beta}\right] \text { and } \phi=\gamma c+\delta-\beta\left[\frac{\gamma c+\delta}{\beta}\right] .
$$

Then $0 \leq \phi<\beta$, which implies that the matrix $B^{\prime}=\left(\begin{array}{ll}\beta & 0 \\ \phi & \gamma\end{array}\right)$ is row-balanced. Moreover, $\operatorname{det}\left(B^{\prime}\right)=D$.

2. $\gamma=0$. Since

$$
\left(\begin{array}{ll}
\alpha & \beta \\
0 & \delta
\end{array}\right)=\left(\begin{array}{ll}
0 & 1 \\
1 & 0
\end{array}\right)\left(\begin{array}{ll}
0 & \delta \\
\alpha & \beta
\end{array}\right)
$$


we have

$$
\left(\begin{array}{cc}
\alpha & \beta \\
0 & \delta
\end{array}\right)\left(\begin{array}{cc}
c+D f_{k} & 1 \\
1 & 0
\end{array}\right)=\left(\begin{array}{cc}
f_{k}^{\prime} & 1 \\
1 & 0
\end{array}\right)\left(\begin{array}{cc}
\delta & 0 \\
\phi^{\prime} & \alpha
\end{array}\right)
$$

with

$$
f_{k}^{\prime}=\alpha^{2} f_{k}+\left[\frac{\alpha c+\beta}{\delta}\right] \text { and } \quad \phi^{\prime}=\alpha c+\beta-\delta\left[\frac{\alpha c+\beta}{\delta}\right]
$$

Then $B^{\prime}=\left(\begin{array}{cc}\delta & 0 \\ \phi^{\prime} & \alpha\end{array}\right)$ is row-balanced and $\operatorname{det}\left(B^{\prime}\right)=D$.

3. $(\alpha, \gamma)=1$. It is well known that $\alpha / \gamma$ has two continued fraction expansions: one ends with a partial quotient $a \geq 2$ and the other one is obtained by replacing $a$ by $a-1,1$. Consider the continued fraction expansion of $\alpha / \gamma$ of even length if $\alpha \delta-\beta \gamma=D$ or of odd length if $\alpha \delta-\beta \gamma=-D$. The product of matrices of the form $\left(\begin{array}{cc}a_{i} & 1 \\ 1 & 0\end{array}\right)$ corresponding to this continued fraction expansion is equal to $\left(\begin{array}{ll}\alpha & \alpha^{\prime} \\ \gamma & \gamma^{\prime}\end{array}\right)$. Therefore, there exist two positive integers $\alpha^{\prime}$ and $\gamma^{\prime}$ such that

$$
\alpha^{\prime}<\alpha, \quad \gamma^{\prime}<\gamma \quad \text { and } \quad \alpha \gamma^{\prime}-\alpha^{\prime} \gamma=\frac{\alpha \delta-\beta \gamma}{D}=u= \pm 1 .
$$

Hence

$$
\left(\begin{array}{ll}
\alpha & \beta \\
\gamma & \delta
\end{array}\right)\left(\begin{array}{cc}
c+D f_{k} & 1 \\
1 & 0
\end{array}\right)=\left(\begin{array}{cc}
\alpha & \alpha^{\prime} \\
\gamma & \gamma^{\prime}
\end{array}\right)\left(\begin{array}{cc}
f_{k}+c^{\prime} & 1 \\
1 & 0
\end{array}\right)\left(\begin{array}{cc}
D & 0 \\
\varepsilon & 1
\end{array}\right) .
$$

$c^{\prime}$ and $\varepsilon$ are defined as follows: since $\alpha \gamma^{\prime}-\alpha^{\prime} \gamma=u$, one has

$$
\alpha^{\prime} \delta-\beta \gamma^{\prime}=\alpha^{\prime} \delta-\beta \frac{u+\alpha^{\prime} \gamma}{\alpha}=\frac{\alpha^{\prime}(\alpha \delta-\beta \gamma)-u \beta}{\alpha},
$$

which implies that $\left|\alpha^{\prime} \delta-\beta \gamma^{\prime}\right| \leq D<c$. Then $c^{\prime}$ and $\varepsilon$ are given by the Euclidean division

$$
c-u\left(\alpha^{\prime} \delta-\beta \gamma^{\prime}\right)=D c^{\prime}+\varepsilon \quad \text { with } 0 \leq \varepsilon<D .
$$

Therefore, the matrix $B^{\prime}=\left(\begin{array}{cc}D & 0 \\ \varepsilon & 1\end{array}\right)$ is row-balanced and $\operatorname{det}\left(B^{\prime}\right)=D$. Then

4. Finally, suppose that $(\alpha, \gamma)=m>1$. Put $\alpha=m \alpha_{1}$ and $\gamma=m \gamma_{1}$.

$$
\left(\begin{array}{ll}
\alpha & \beta \\
\gamma & \delta
\end{array}\right)=\left(\begin{array}{ll}
\alpha_{1} & \beta \\
\gamma_{1} & \delta
\end{array}\right)\left(\begin{array}{cc}
m & 0 \\
0 & 1
\end{array}\right)
$$

The transformation of

$$
\left(\begin{array}{ll}
\alpha & \beta \\
\gamma & \delta
\end{array}\right)\left(\begin{array}{cc}
c+D f_{k} & 1 \\
1 & 0
\end{array}\right)
$$

is given by two successive transformations described in 2 and 3 .

In all cases $f_{k}^{\prime}$ is the image of $c+D f_{k}$ by a map of the form $x \mapsto(u x+v) / w$ with integral coefficients $(u, w \neq 0)$. 
Lemma 3. Let $\xi=\left[c_{0} ; c_{1}, \ldots, c_{n-1}, \overline{C_{1}(k), \ldots, C_{p}(k)}\right]_{k=0}^{\infty}$ be in $\mathcal{R}$ with the relation

$$
\left(\begin{array}{c}
C_{p}(k+1) \\
C_{p-1}(k+1) \\
\vdots \\
C_{1}(k+1)
\end{array}\right)=M\left(\begin{array}{c}
C_{p}(k) \\
C_{p-1}(k) \\
\vdots \\
C_{1}(k)
\end{array}\right)
$$

for all integers $k \geq 0$. Let $g_{1}, \ldots, g_{p}$ be $p$ maps of the form $g_{j}: x \mapsto$ $\left(u_{j} x+v_{j}\right) / w_{j}$ with integral coefficients such that $C_{j}^{\prime}(k)=g_{j}\left(C_{j}(k)\right)$ is a positive integer for all integers $j(1 \leq j \leq p)$ and all integers $k \geq 0$. Then $\xi^{\prime}=\left[c_{0}^{\prime} ; c_{1}^{\prime}, \ldots, c_{n^{\prime}-1}^{\prime}, \overline{C_{1}^{\prime}(k), \ldots, C_{p}^{\prime}(k)}\right]_{k=0}^{\infty} \in \mathcal{R}$.

Proof. Let $P$ defined by

$$
P(x)=x^{p^{\prime}}-\sum_{i=0}^{p^{\prime}-1} \alpha_{i} x^{i}
$$

be the minimal polynomial of $M$. Then

$$
C_{j}\left(k+p^{\prime}\right)=\sum_{i=0}^{p^{\prime}-1} \alpha_{i} C_{j}(k+i) \quad \text { for all integers } j\left(0 \leq j \leq p^{\prime}-1\right) .
$$

Therefore, there exists an integer $\beta_{j}$ such that

$$
C_{j}^{\prime}\left(k+p^{\prime}\right)=\sum_{i=0}^{p-1} \alpha_{i} C_{j}^{\prime}(k+i)+\beta_{j}
$$

Hence

$$
C_{j}^{\prime}\left(k+2 p^{\prime}\right)=C_{j}^{\prime}\left(k+p^{\prime}\right)+\sum_{i=0}^{p^{\prime}-1} \alpha_{i}\left(C_{j}^{\prime}\left(k+p^{\prime}+i\right)-C_{j}^{\prime}(k+i)\right)
$$

and $\xi^{\prime} \in \mathcal{R}$.

Now, we are ready to give the proof of Theorem 2. Let $\xi$ be defined as in Lemma 1 and compute $\xi^{\prime}=(a \xi+b) /(c \xi+d)$ with the transducer $\mathcal{T}_{D}$. Denote $B=B_{r}=B_{r+q}$. If $A_{1}(0)=A_{1}(1)=c$, then of course in both cases we obtain the same results $\phi_{c}(B)$ and $\psi_{c}(B)$. If $A_{1}(0) \neq A_{1}(1)$, then there exists an integer $c>D$ and two nonnegative integers $f_{0}$ and $f_{1}$ such that $A_{1}(0)=c+D f_{0}$ and $A_{1}(1)=c+D f_{1}$. Then by Lemma $2, \phi_{A_{1}(0)}(B)=$ $\phi_{A_{1}(1)}(B)$. Now, $\psi_{A_{1}(0)}(B)$ and $\psi_{A_{1}(1)}(B)$ are composed of two subwords. The first subwords are the same (and are possibly empty). They correspond to the matrix $C$ of Lemma 2. The last subwords contain only one letter. There exists a map $g: x \mapsto(u x+v) / w(u, w \neq 0)$ with integral coefficients such that the last letter of $\psi_{A_{1}(0)}(B)$ is $g\left(A_{1}(0)\right)$ (respectively $\psi_{A_{1}(1)}(B)$ and $\left.g\left(A_{1}(1)\right)\right)$. 
By iteration, $\phi_{A_{i}(0)}\left(B_{r+i-1}\right)=\phi_{A_{i}(1)}\left(B_{r+i-1}\right)$ for $i=1, \ldots, q$ and the sequence of states $\left(B_{m}\right)_{m \in \mathbb{N}}$ is ultimately periodic. Before cancelling the "zeros" with the contraction map $\mu$, we obtain $\xi^{\prime}=\left[c_{0}^{\prime} ; c_{1}^{\prime}, \ldots, c_{n^{\prime}-1}^{\prime}\right.$, $\left.\overline{A_{1}^{\prime}(k), \ldots, A_{q^{\prime}}^{\prime}(k)}\right]_{k=0}^{\infty}$ : if $A_{j^{\prime}}^{\prime}(k)$ is not a constant sequence, then there exists an index $j$ and three integers $u_{j}, v_{j}$ and $w_{j} \quad\left(u_{j}, w_{j} \neq 0\right)$ such that $A_{j^{\prime}}^{\prime}(k)=\left(u_{j} A_{j}(k)+v_{j}\right) / w_{j}$ for all integers $k \geq 0$. By Lemma $3, \xi^{\prime} \in \mathcal{R}$.

Notice that in case $\xi$ is Hurwitzian, $\xi^{\prime}$ is also Hurwitzian and the nonconstant polynomials in the quasi-periods of $\xi$ and $\xi^{\prime}$ have the same degrees.

3. Example. Let $\left(F_{n}\right)_{n \in \mathbb{N}}$ be the Fibonacci sequence which can be defined by

$$
\left(\begin{array}{cc}
F_{n+1} & F_{n} \\
F_{n} & F_{n-1}
\end{array}\right)=\left(\begin{array}{ll}
1 & 1 \\
1 & 0
\end{array}\right)^{n}
$$

Denote by $\xi$ the real number $\xi=\left[\overline{F_{n}}\right]_{n=1}^{\infty}$ which is in $\mathcal{R}$. Now compute the continued fraction expansion of $2 \xi$, using the transducer of the multiplication by 2 (see [5]) which contains five states.

The initial state of the transducer is $B_{0}=A=\left(\begin{array}{ll}2 & 0 \\ 0 & 1\end{array}\right)$. The continued fraction expansion of $\xi$ can be written

$$
\xi=\left[1 ; 1,2,3, \overline{F_{6 k+5}, F_{6 k+6}, F_{6 k+7}, F_{6 k+8}, F_{6 k+9}, F_{6 k+10}}\right]_{k=0}^{\infty} .
$$

A simple computation leads to $B_{10}=B_{4}=\left(\begin{array}{ll}2 & 0 \\ 0 & 1\end{array}\right), F_{n} \geq 2$ for $n \geq 3$ and $F_{n}=F_{n+6} \bmod 2$ for all integers $n$.

Hence, by applying the transducer $\mathcal{T}_{2}$ and the contraction map $\mu$, one has:

Proposition.

$$
2 \xi=\frac{[3 ; 2,1,1,}{\left.2 F_{6 k+5}, \frac{F_{6 k+6}}{2}, 2 F_{6 k+7}, \frac{F_{6 k+8}-1}{2}, 1,1, \frac{F_{6 k+9}}{2}-1,1,1, \frac{F_{6 k+10}-1}{2}\right]_{k=0}^{\infty} .}
$$

It is clear that $2 \xi \in \mathcal{R}$.

\section{References}

[1] H. Cabannes, Étude des fractions continues ayant leurs quotients en progression arithmétique ou en progression géométrique, La Revue Scientifique 83 (1945), 230233.

[2] C. S. Davis, On some simple continued fractions connected with e, J. London Math. Soc. 20 (1945), 194-198.

[3] L. Euler, De fractionibus continuis dissertatio, Comment. Acad. Petropol. 9 (1744), 115-127. 
[4] D. H. Lehmer, Continued fractions containing arithmetic progressions, Scripta Math. 29 (1973), 17-24.

[5] P. Liardet et P. Stambul, Transducteurs et fractions continuées, preprint, 1996.

[6] K. R. Matthews and R. F. C. Walters, Some properties of the continued fraction expansion of $\frac{m}{n} e^{1 / q}$, Proc. Cambridge Philos. Soc. 67 (1970), 67-74.

[7] O. Perron, Die Lehre von den Kettenbrüchen, Bd. 1, 3rd ed., Teubner, 1954, 110138.

[8] A. J. van der Poorten, An introduction to continued fractions, in: J. H. Loxton and A. J. van der Poorten (eds.), Diophantine Analysis, Cambridge University Press, 1986, 99-138.

[9] G. Raney, On continued fractions and finite automata, Math. Ann. 206 (1973), 265-283.

[10] P. Stambul, Contribution à l'étude des propriétés arithmétiques des fractions continuées, Thèse de l'Université de Provence, 1994.

Centre de Mathématiques et Informatique

DSA

Université de Provence

39, rue Joliot Curie

F-13543 Marseille Cedex 13, France

E-mail: liardet@gyptis.univ-mrs.fr

Received on 11.2.1996

and in revised form on 14.6.1996 